\title{
CLPDI Algorithm in UWB Synchronization
}

\author{
Simone Soderi, Jari Iinatti, Matti Hämäläinen \\ Centre for Wireless Communications (CWC), University of Oulu, P.O.Box 4500, \\ FIN-90014 University of Oulu, Finland \\ emails: s.soderi@inwind.it, \{jari.iinatti,matti.hamalainen\}@ee.oulu.fi
}

\begin{abstract}
This paper studies code synchronization in time hopping (TH) ultra wideband (UWB) systems. In TH UWB systems, narrow pulses based on Gaussian pulse are positioned in different locations in consecutive time frames. The location of each frame is defined by a spreading code. The receiver collects the energy of the pulses using the same spreading code. To be able to do that, it has to synchronize itself to the incoming signal. Due to the large bandwidth of the signal, several replicas of the signals arrive into the receiver. Thus, the energy of the signal is spread in time domain causing extra problems to the synchronization procedure. In this paper, earlierly proposed method for direct sequence spread spectrum system is applied for time hopping system. The method is simulated in a multipath environment. The performance measure used is the mean acquisition time. Constant false alarm rate criterion is used for the threshold setting rule for comparator. The energies of the multipath components are combined in the acquisition process. Therefore, diversity combining is obtained in the acquisition process, which also leads to performance improvement.
\end{abstract}

\section{INTRODUCTION}

The most important task in a communication system receiver is synchronization that can be divided into several levels: carrier, symbol, bit, frame, and network [1]. Spread spectrum systems, like code division multiple access (CDMA) and $U W B$-systems, need in addition to those code synchronization in which the received spreading code and receiver used spreading code are forced to the same phase. Otherwise it is not possible to despread the signal at the receiver, and thus the data detection doesn't succeed. Code synchronization is performed in two steps: acquisition (initial synchronization) and tracking (fine synchro- nization). This work discusses the first one in UWB system.

UWB systems use very large bandwidths, and therefore, received signal includes dense multipath profile having many components [2]. From the point of view of the code synchronisation, this phenonem causes problems: the energy of the signal is spread over several paths, and the energy of each path is very low. Therefore, the paths are difficult to acquire. Depending on the receiver structure, a number of paths should be acquired.

The UWB system described here employs Time-Hopping $(T H)$ baseband signal composed of very short pulses. Data modulation is neglegted. Recently, an algorithm called chip level post detection integration (CLPDI) was proposed for code acquisition in direct sequence CDMA systems [3]. The method is suitable for synchronisation in multipath environments. In this work, the method is applied for time hopping UWB system due to nature of UWB signals and performance is evaluated by aid of computer simulations.

The paper is organised as follows. In section 2, TH-UWB system model and methods for synchronization are presented. Section 3 describes the simulation model. Section 4 gives the simulation results and conclusions are given in Section 5.

\section{SYSTEM AND SYNCHRONISATION MODEL}

\subsection{Time Hopping UWB System}

Time hopping UWB systems are generally based on impulse radio concept [4]. The idea of a simple TH-UWB concept is presented in Fig. 1 , where the pulse width is expressed as $T_{p}$ and pulse repetition interval (time hopping frame) 
as $T_{f}$. The time hopping instants depend on the pseudo random (PR) time hopping code. Time gaps between the consecutive pulses are also random due to the PR code. The length of the PR code ( $M$ code phases) is in general independent of $N$, which defines processing gain of the system by introducing the number of pulses used to transmit one data bit. In TH mode the duty cycle $T_{p} / T_{f}<100 \%$. The data rate is inversely proportional to the number of pulses used to transmit one symbol and also inversely proportional to the frame length. Bit rate $R_{b}$ of TH-UWB system can be defined by

$$
R_{b}=\frac{1}{T_{b}}=\frac{1}{N T_{f}} .
$$

In TH systems, users are separated using different PR codes. In a frame, there are $M$ possible transmission instants, so $M$ users can be allocated into the system without creating interference in ideal conditions.

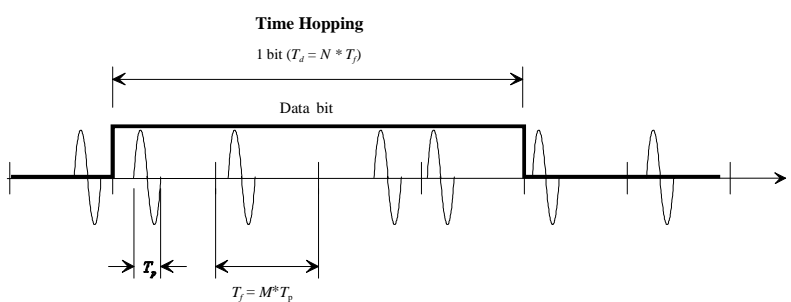

Figure 1. The idea of time hopping system concept.

In time hopping mode (TH-UWB), the modulated information signal $s(t)$ for the $m^{t h}$ user can be presented as [5]

$$
s^{(m)}(t)=\sum_{k=0}^{\infty} \sum_{j=0}^{N} w\left(t-k T_{b}-j T_{f}-\left(c_{m}\right)_{j}^{(m)} T_{c}\right) d_{k}^{(m)}(2)
$$

where $d_{k}$ is the $k^{\text {th }}$ data bit (always 1 in this paper), $\left(c_{w}\right)_{j}$ is the $j^{\text {th }}$ code phase defined by the spreading code $c(t)$ and $w(t)$ is the pulse waveform. The pulse waveform defines the frequency band of the signal. Modifications of the basic Gaussian pulse are examples of the suitable waveforms and their effects have been studied, e.g., in [5]. $T_{c}$ is chip length and bit length is $T_{b}=N T_{f}$. In TH-UWB $T_{f} \gg T_{p}$ producing a low duty cycle. In the following, $M$ is assumed to be same as $N$. The input signal for the receiver in one user case is given by

$r(t)=\sum_{l=1}^{L} A_{l} \cdot s^{(1)}\left(t-\tau_{l}\right)+n(t)$

where $A_{l}$ and $\tau_{l}$ are amplitude and delay of path $l$, respectively, $L$ is the number of resolvable multipaths and $n(t)$ is additive white Gaussian noise. The delay can be presented as a portion of $T_{c}$ as $\tau_{l}=\xi T_{c}$. In the received signal waveform in $s(t)$, the effect of antenna should be taken into account.

\subsection{Synchronization in UWB System}

The synchronization stage must provide an estimate $\xi_{e}$ for the timinig offset $\xi$ (i.e., $\tau_{e}$ ). Maximum Likelihood (ML) algorithm generates several values $\xi_{e}$ to evaluate when conditional propability density function of the received signal $p(r \mid \tau)$ gets the maximum value [6]. Basically

$\tau_{e}=\arg \max _{\tau_{e}} p\left(r \mid \tau_{e}\right)$

If we have white Gaussian noise, the conditional probability density function is easy to evaluate, and for the optimal estimation $\tau_{e}$, we have to maximize

$$
\begin{aligned}
\lambda\left(\tau_{e}\right)= & \int_{0}^{T_{o}} r(t) s\left(t, \tau_{e}\right) d t \\
& =\int_{0}^{T_{o}} A_{l} \sum_{k=0}^{\infty} \sum_{j=1}^{N} w\left(t-k T_{b}-j T_{f}-\tau\right) . \\
& \sum_{k=0}^{\infty} \sum_{j=1}^{N} w\left(t-k T_{b}-j T_{f}-\tau_{e}\right) d t+n_{c}(t)
\end{aligned}
$$

where $n_{c}(t)$ is noise component. In practice, the algorithm is too complex and some approximations have to be used. In so-called serial search strategy, the phase of the local code is changed step by step in equal increments. In this way the correct position within the uncertainity region could be found. Synchronization methods for spread spectrum systems can be found from [7]. 


\subsection{Synchronisation Schemes}

The synchronisation algorithm for multipath environment proposed for DS CDMA is presented in Fig. 2 [3]. In DS systems, the impulse response of the matched filter (MF) is the timereversed replica on the spreading code, i.e., it has coefficients given by the spreading code and delay between concecutive coefficients are $T_{c}$. The output signal of the MF is proportional to the autocorrelation function (ACF) of the spreading code. The sampling at the output of the MF is made at least at the chip rate. The MF is followed by a threshold comparison, and if the threshold $T_{h}$ is crossed, the acquisition process ends. If the threshold is crossed by the $\mathrm{ACF}$ at the zero delay, the result is detection, which occurs with probability of detection, $P_{d}$. If the threshold is crossed with some other delay, a false alarm occurs. This happens with the probability of false alarm, $P_{f a}$. False alarms may be catastrophic, and cause total miss of the correct code phase. Because the MF gives its peak when the code is inside the filter, a multipath channel leads to several peaks.

Chip Level Post Detection Integration (CLPDI) performs post detection integration at the chip level, i.e., a number of consecutive samples at the output of the MF are combined. CLPDI is performed over $m$ samples and CLPDI output is sampled at multiples of $m T_{c}$ as is presented in Fig. 3. Because the sampling is done as multiples of $m T_{c}$, consecutive samples at the output of CLPDI are uncorrelated, and the uncertainty region, i.e., number of cells to be tested in the acquisition is decreased compared to pure MF acquisition. The number of cells in the uncertainty region is now reduced to $N_{m}=N / m$; also the number of correct cells is reduced. These have affect on the performance.

In time hopping system, the MF collects the samples together according to delays between concecutive chips (pulses as presented in Fig. 1 ), i.e., the spreading code sets the delays between concecutive " 1 "s in the impulse response. Therefore MF waits until chips (pulses) are coming in pre-determined time slots inside frames.

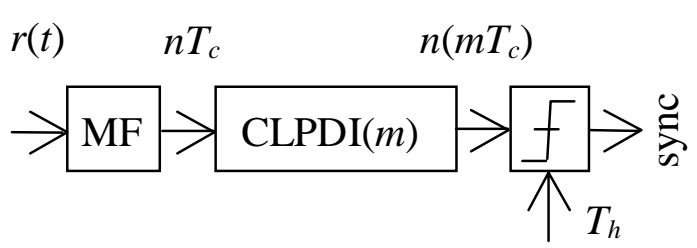

Figure 2. MF with CLPDI.

\subsection{Performance Measures}

Mean acquisition time is used as a performance measure. It can be calculated for the CLPDI case as [3]

$$
\begin{aligned}
& T_{M A}=\frac{P_{M}^{L / m}\left[L T_{c}+(N-L)\left(T_{c}+T_{f a} P_{f a}\right)\right]}{1-P_{M}^{L / m}} \\
& +\frac{\left[N T_{c}+(N-L)\left(T_{c}+T_{f a} P_{f a}\right)\right]^{L / m-1} i P_{M}^{i}}{N \sum_{i=0}^{L / m-1} P_{M}^{i} / m}+m T_{c}(6) \\
& +\frac{(N-L)(N-L+m)\left(T_{c}+T_{f a} P_{f a}\right)}{2 N}+N T_{c}
\end{aligned}
$$

where the uncertainty region $N$ is the length of the code, and no a priori information of the correct code phase exists at the beginning of acquisition. $P_{f a}$ is the probability of false alarm at the output of the CLPDI, $P_{M}=1-P_{d}$ where $P_{d}$ is the probability of detection in the correct code phase, $T_{f a}=K_{p} T_{c}$ is the penalty time caused by a false alarm, and $T_{c}$ is chip interval. The probabilities of detection and false alarms are measured by simulations and verified in test cases by the theoretical values. On the other hand in a multipath propagation environment without the CLPDI block the $T_{M A}$ is evaluated with a similar equation ([8])

\section{SIMULATION MODEL}

TH UWB simulation model with matched filter synchronization is presented in Fig. 3, where the outputs in different position of the system are also described. 


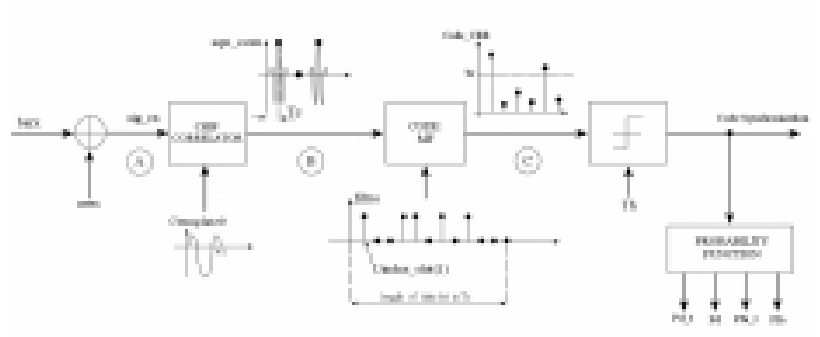

Figure 3. Simulation model for TH-UWB synchronizer in AWGN channel.

Chip correlation (point A in Fig. 3) is performed by correlating the received signal with template waveform having the same shape than the received signal (after antenna). In the simulations, the pulse waveform in the transmitter is Gaussian pulse. Therefore, the reference pulse in the receiver is the second derivative of the Gaussian pulse due to effect of the transmitting and receiving antenna [9]. To reduce the complexity in the simulations, only one sample per $T_{c}$ is used. This can be done when the pulse width $\left(T_{p}\right)$ is smaller than chip length $\left(T_{c}\right)$ and chip synchronism is assumed. The output of the chip correlator is a sequence that contains the information about the pulse locations within the received sequence in every frame. The chip correlator output is fed into a code matched filter CMF. The filter is matched to the whole spreading code (point B in the Fig. 3). In the TH system it means, that delays between consecutive " 1 "s in impulse respense is not fixed but dependents on the code phase as seen in Fig. 4. After the CMF, a threshold comparison is used (point $\mathrm{C}$ in the Fig. 3). Fig. 4 presents an example of the output of code matched filter and describes also the correct synchronization points.

The Constant False Alarm Rate criterion (CFAR) is used to set the threshold for the comparator, which means that $P_{f a}$ is the same for every false code phases. To evaluate the performance of the model, the theoretical values of $P_{d}$ and $P_{f a}$ are compared with the simulated ones. After the CMF, the samples have a Gaussian distribution, and $P_{d}$ and $P_{f a}$ are given by [1]

$$
P_{d}=\frac{1}{2}-\frac{1}{2} \operatorname{erf}\left(\frac{T h-A}{\sqrt{2} \sigma}\right)
$$

$P_{f a}=\frac{1}{2}-\frac{1}{2} \operatorname{erf}\left(\frac{T h}{\sqrt{2} \sigma}\right)$

where $A$ is output when code phase is correct and $\sigma$ is standard deviation of the noise at the output of CMF which can be evaluated for the test cases. Theoretical values were compared with the simulated values to ensure the correctness of the model.

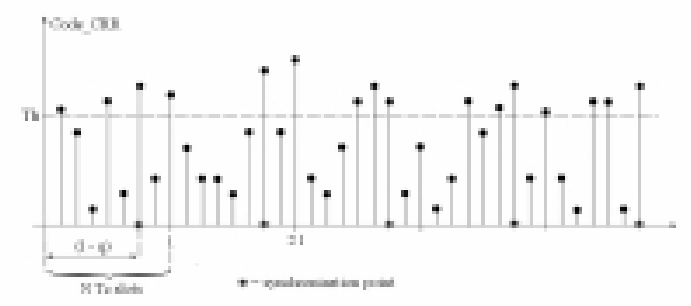

Figure 4. Output of the code matched filter when six bits are transmitted.

In the CLPDI simulations, the CLPDI block is inserted after the code MF in the block diagram of the TH-UWB synchronizer (Fig. 3).

\section{SIMULATION RESULTS}

A channel with static multipath is considered. The paths have equal energies because it has been found to be the worst case from the acquisition point of view in static channel [8]. One sample per chip is taken to achieve lower simulation times and chip synchronism is assumed. We use a code length $N=80$ and penalty time $T_{f a}=100 T_{b}$. Constant false alarm rate criterion (CFAR) is used when setting the threshold of the comparator $\left(P_{f a}=10^{-2}\right)$. Proper $T_{h}$ was searched before simulations, so that the required $P_{f a}$ was obtained for each SNR values. Simulations were performed over 100 bits, i.e., the number of the correct code phases is $100 \mathrm{~L}$ and the number of false code phases is $100(80-$ $L)$.

Figs. 5 and 6 present $T_{M A}$ performance of the synchronizer with and without CLPDI for 2 and 4 path channels, respectively. In a 4 path channel, $m$ in CLPDI is either 2 or 4. $P_{d}$ and $P_{f a}$ are obtained by simulation and $T_{M A}$ is calculated from (6). $T_{M A}$ is also presented for a one path channel. In TH-UWB systems, CLPDI of- 
fers an improvement in the performance compared to conventional MF synchronizer due to reduced number of total cells to be examined and due to increased $P_{d}$ in correct cells. In a 4 path channel, performance improvement is obtained using both $m$ values 2 and 4. Similar behaviour is observed as in WCDMA systems [3].

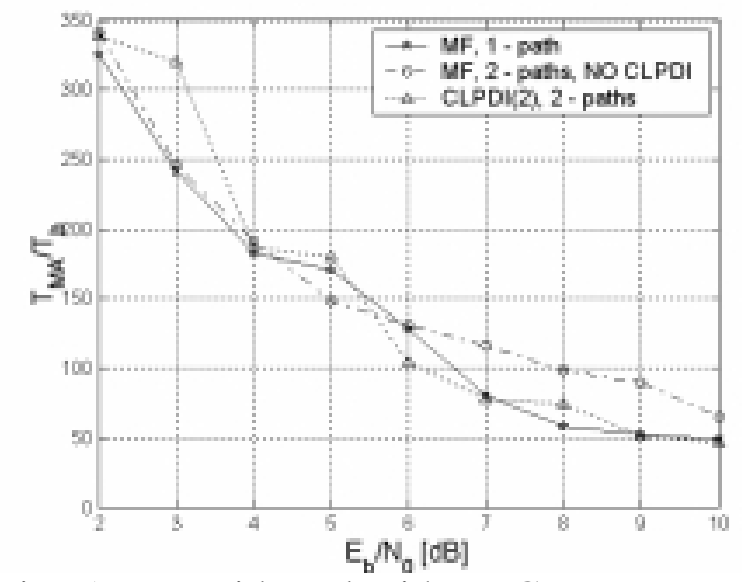

Fig. 5. $T_{M A}$ with and without CLPDI, $N=80$, $L=2, T_{b}=N T_{c}, T_{f a}=100, P_{f a}=10^{-2}$.

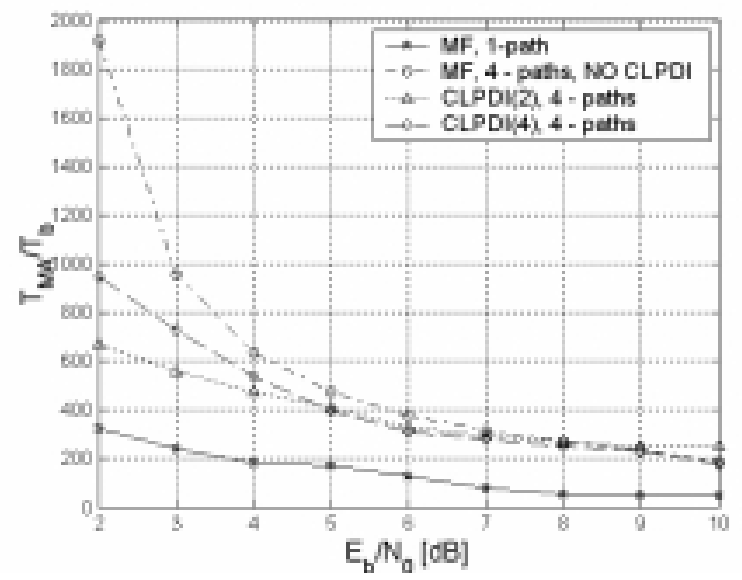

Fig. 6. $T_{M A}$ with and without CLPDI, $N=80$, $L=4, T_{b}=N T_{c}, T_{f a}=100, P_{f a}=10^{-2}$.

\section{CONCLUSIONS}

A chip level post detection integration code synchronization method was applied to a time hopping UWB system in this paper. The task of the method is to utilize dense multipath propagation of UWB environment during code acquisition process. The method increases the probability of detection of the burst of multipath components, i.e., it decreases the mean acquisition time for finding the existence of multipath profile. The simulation results indicate that the method improves the acquisition performance and is therefore, usable in UWB communication system.

\section{ACKNOWLEDGEMENTS}

This study has been financed by the National Technology Agency of Finland (Tekes), Nokia, Elektrobit and the Finnish Defence Forces. Authors would like to thank the sponsors for their interest and support. This work is also financed by European Commission via Ultrawaves project (IST-2001-35189). Authors would also like to thank Dr. Ian Oppermann for the valuable comments concerning the paper. Mr. Raffaello Tesi and Mr. Veikko Hovinen from CWC are appreciated for their contribution during the simulator development work.

\section{REFERENCES}

[1] J. Iinatti, Matched Filter Code Acquisition Employing a Median Filter in Direct Sequence Spread-Spectrum Systems with Jamming, Acta Universitatis Ouluensis C102, Doctoral thesis, University of Oulu, Finland, 1997.

[2] V. Hovinen, M. Hämäläinen, T. Pätsi, Ultra Wideband Indoor Radio Channel Models: Preliminary Results, In Proc. of UWBST2002, Baltimore, USA.

[3] J. Iinatti, M. Latva-aho, A Modified CLPDI for Code Acquisition in Multipath Channel, In Proc. of PIMRC2001, San Diego, USA.

[4] R.A. Scholtz, M.Z. Win, Impulse Radio, In Proc. of PIMRC97, Helsinki, 1997.

[5] M. Hämäläinen, V. Hovinen, R. Tesi, J. Iinatti, M. Latvaaho, On the UWB System Coexistence with GSM900, UMTS/WCDMA, and GPS Systems, IEEE Journal on Selected Areas in Communications, Dec.2002, Vol. 20, No. 9, pp. 1712-1721..

[6] S. Glisic, B. Vucetic, Spread Spectrum CDMA Systems for Wireless Communications Artech House Publisher, 1997.

[7] M. Katz, Code Acquisition in Advanced CDMA Networks, Acta Universitatis Ouluensis C175, Doctoral thesis, University of Oulu, Finland, 2002.

[8] J. Iinatti, Performance of DS Code Acquisition in Static and Fading Multi-path Channel, IEE Proceedings - Communications, vol.147, Issue 6, December 2000, pp.355360.

[9] F. Ramirez-Mireles, R. A. Scholtz, System Performance Analysis of Impulse Radio Modulation," In Proc. of RAWCON'98, Colorado Springs, CO, USA. 